\title{
Multidecadal Fluctuation of the Wintertime Arctic Oscillation Pattern and Its Implication
}

\author{
Hainan Gong, Lin Wang, Wen Chen, And Debashis Nath \\ Center for Monsoon System Research, and LASG, Institute of Atmospheric Physics, Chinese Academy of Sciences, \\ Joint Center for Global Change Studies, and College of Earth and Planetary Sciences, \\ University of Chinese Academy of Sciences, Beijing, China
}

(Manuscript received 8 August 2017, in final form 31 March 2018)

\begin{abstract}
The multidecadal fluctuations in the patterns and teleconnections of the winter mean Arctic Oscillation (AO) are investigated based on observational and reanalysis datasets. Results show that the Atlantic center of the AO pattern remains unchanged throughout the period 1920-2010, whereas the Pacific center of the AO is strong during 1920-59 and 1986-2010 and weak during 1960-85. Consequently, the link between the AO and the surface air temperature over western North America is strong during 1920-59 and 1986-2010 and weak during 1960-85. The time-varying Pacific center of the AO motivates a revisit to the nature of the AO from the perspective of decadal change. It reveals that the North Pacific mode (NPM) and North Atlantic Oscillation (NAO) are the inherent regional atmospheric modes over the North Pacific and North Atlantic, respectively. Their patterns over the North Pacific and North Atlantic remain stable and change little with time during 1920-2010. The Atlantic center of the AO always resembles the NAO over the North Atlantic, but the Pacific center of the AO only resembles the NPM over the North Pacific when the NPM-NAO coupling is strong. These results suggest that the AO seems to be fundamentally rooted in the variability over the North Atlantic and that the annular structure of the AO very likely arises from the coupling of the atmospheric modes between the North Pacific and North Atlantic.
\end{abstract}

\section{Introduction}

The Arctic Oscillation (AO) proposed by Thompson and Wallace (1998) is defined as the first empirical orthogonal function (EOF) mode of the sea level pressure (SLP) in the extratropical Northern Hemisphere. It reflects a seesaw in the SLP field between the Arctic and midlatitudes. In this sense, the AO is also referred to as the northern annular mode (Thompson and Wallace 2001). Previous studies have demonstrated that the AO can strongly influence the global and regional climate through its teleconnections (e.g., Thompson and Wallace 2001; Gong et al. 2001; Chen and Kang 2006; Tan et al. 2008; Wang and Chen 2010; Chen et al. 2013). However, the physical significance of the mode, especially the Pacific center of the AO pattern, has been a matter of great debate since the AO was proposed (e.g., Deser 2000; Wallace 2000; Ambaum et al. 2001; Christiansen 2002; Rogers and McHugh 2002; Wallace and Thompson 2002; Feldstein and Franzke 2006; Itoh 2008). Thompson and Wallace

\footnotetext{
Corresponding author: Dr. Lin Wang, wanglin@mail.iap.ac.cn
}

(1998) suggested that the AO is a fundamental mode of the climate variability that describes the seesaw of air masses between the Arctic and the midlatitudes. Deser (2000) found that among the three centers of the AO only the Arctic and Atlantic centers correlate strongly with each other, whereas the Pacific center is weakly correlated with the other two. Wallace and Thompson (2002) explained that the correlation between the Pacific and Atlantic centers becomes strong when the second EOF mode of the SLP is linearly removed from the original SLP field and that the $\mathrm{AO}$ is a reliable physical mode. In contrast, Ambaum et al. (2001) considered a three-point seesaw system representing three centers of action: the Arctic, Euro-Atlantic, and Pacific regions. They suspected that the North Pacific variability, together with the North Atlantic Oscillation (NAO), constitutes the AO and that the Pacific center of the AO may be produced from the North Pacific variability. In fact, the discussion about the nature of the AO still continues even in recent years (e.g., Itoh 2008; Sun and Tan 2013).

From another aspect, some studies found that the spatial structure of the $\mathrm{AO}$ is nonstationary and that the 
Pacific center of the AO cannot be observed very often (e.g., Honda and Nakamura 2001; Castanheira and Graf 2003; Zhao and Moore 2009; Sun and Tan 2013; Shi and Nakamura 2014; Dai and Tan 2017). For example, Honda and Nakamura (2001) pointed out that the amplitude of the Pacific AO pattern is linked to the Aleutian low and the Icelandic low seesaw (AIS) pattern in late winter (February-March) during 1973-94. During the twentieth century, the leading mode of SLP variability over the Northern Hemisphere is remarkably different in different phases of the AIS (Shi and Nakamura 2014). Besides, the Pacific center of the AO pattern can also be modulated significantly by the stratospheric polar vortex. It is strong and clear when the stratospheric polar vortex is strong, and it is weak and unclear when the stratospheric polar vortex is weak (Castanheira and Graf 2003; Sun and Tan 2013; Tan et al. 2015). These results indicate that the spatial structure of the AO pattern is variable under different conditions.

The above studies show the nonstationary feature of the spatial structure of the AO pattern, but their results were obtained under certain specific conditions (e.g., the extreme phases of the AIS pattern or the strength of the stratospheric polar vortex). However, it remains unknown how the wintertime AO pattern itself changes with time. It is also not clear whether the teleconnection of the wintertime AO changed during the twentieth century. Therefore, in this study we will investigate the possible changes in the pattern and teleconnections of the wintertime AO based on long-term observational and reanalysis data. Moreover, we will revisit the discussion on the nature of the $\mathrm{AO}$ from the perspective of the decadal changes of the wintertime AO pattern. The rest of the paper is organized as follows: section 2 describes the data and methods. Section 3 presents the observational evidence for the time-varying pattern and teleconnections of the wintertime AO during the twentieth century. Section 4 revisits the discussion of the nature of the AO from perspective of decadal change. Finally, a summary is presented in section 5 .

\section{Data and methods}

The monthly mean SLP (HadSLP2r) and surface air temperature (SAT) anomalies data (HadCRUT4) are from the Met Office Hadley Centre (Allan and Ansell 2006; Jones et al. 2012). The horizontal resolution of HadSLP2r and HadCRUT4 is $5^{\circ} \times 5^{\circ}$, and the temporal coverage of the two datasets is from 1850 to the present. To verify the robustness of the results, the monthly mean SLP and SAT data from two reanalysis datasets are also employed. One is the Twentieth Century
Reanalysis dataset, version 2c (20CR-V2c), provided by the National Oceanic and Atmospheric Administration/ Earth System Research Laboratory Physical Sciences Division (NOAA/ESRL PSD), which has $2^{\circ} \times 2^{\circ}$ horizontal resolution and spans from 1851 to the near present (Compo et al. 2011; Giese et al. 2016). The other is the monthly mean ERA-20C dataset provided by the European Centre for Medium-Range Weather Forecasts (ECMWF) (Poli et al. 2016), which covers the period from 1900 to 2010 at the same horizontal resolution of $2^{\circ} \times 2^{\circ}$.

Considering the $\mathrm{AO}$ is the strongest and most active in boreal winter (Thompson and Wallace 2000), this study focus on the winter (December-February) mean AO. Following Thompson and Wallace (1998), the wintertime AO index is defined as the principal component (PC) of the first EOF mode of the winter mean SLP variability in the extratropical Northern Hemisphere $\left(20^{\circ}-90^{\circ} \mathrm{N}\right)$. In addition to the AO, several other wintertime regional modes were also used in this study. One is the dominant atmospheric mode over the North Atlantic, referred to as the NAO (Hurrell 1995), and the other is the dominant atmospheric mode over the North Pacific, referred to as the North Pacific mode (NPM; Gong et al. 2017). These two regional modes are defined as the first EOF modes of the winter mean SLP anomalies over the North Atlantic $\left(20^{\circ}-80^{\circ} \mathrm{N}, 90^{\circ} \mathrm{W}-40^{\circ} \mathrm{E}\right)$ and the North Pacific $\left(20^{\circ}-65^{\circ} \mathrm{N}, 120^{\circ} \mathrm{E}-120^{\circ} \mathrm{W}\right)$, respectively. The corresponding PCs are defined as the NAO and NPM indices (Hurrell et al. 2003; Gong et al. 2017), respectively. In previous studies, several indices such as the Pacific-North America (PNA) pattern index (Wallace and Gutzler 1981) and the North Pacific index (Trenberth and Hurrell 1994) have been proposed to represent the variability of the wintertime North Pacific circulation. Here, we selected the EOF method to define the dominant mode of the wintertime North Pacific variations to keep consistency with the definition used for the NAO. The correlation coefficient between the winter mean NPM index and the PNA (North Pacific) index is $-0.92(0.99)$ during 1920-2010. This suggests that the NPM defined in this study represents almost the same variability described in the other definitions mentioned in the above references. Considering the many abbreviations that are used in this paper, Table 1 is included to summarize all abbreviations used in this study.

Few observations exist before 1920, which causes a large spread among the different datasets (Smoliak and Wallace 2015; Wegmann et al. 2017; Wang et al. 2017), and the ERA-20C dataset is available to 2010 (Compo et al. 2011); therefore, the time period $1920-2010$ is employed in this study, constituting 91 winters from 
TABLE 1. Abbreviations used in the text

\begin{tabular}{|c|c|}
\hline Abbreviation & Explanation \\
\hline $\mathrm{ACI}$ & $\begin{array}{l}\text { Arctic center intensity index, the area-averaged SLP anomalies over the Arctic }\left(55^{\circ}-90^{\circ} \mathrm{N}, 0^{\circ}-360^{\circ}\right) \text { obtained by } \\
\text { regressing winter mean SLP onto a simultaneous index }\end{array}$ \\
\hline $\mathrm{ACI}_{\mathrm{AO}}$ & ACI for AO obtained using $\mathrm{AO}$ index \\
\hline AIS & Aleutian low and the Icelandic low seesaw \\
\hline $\mathrm{AO}$ & $\begin{array}{l}\text { Arctic Oscillation, the first EOF mode of the winter mean SLP over the extratropical Northern Hemisphere }\left(20^{\circ}-90^{\circ} \mathrm{N} \text {, }\right. \\
\left.0^{\circ}-360^{\circ}\right)\end{array}$ \\
\hline EOF & Empirical orthogonal function \\
\hline NACI & $\begin{array}{l}\text { North Atlantic center intensity index, the area-averaged SLP anomalies over the North Atlantic }\left(20^{\circ}-55^{\circ} \mathrm{N} \text {, }\right. \\
\left.80^{\circ} \mathrm{W}-40^{\circ} \mathrm{E} \text {, denoted by the red box in Fig. } 7 \mathrm{a}\right) \text { obtained by regressing winter mean SLP onto a simultaneous index }\end{array}$ \\
\hline $\mathrm{NACI}_{\mathrm{AO}}$ & NACI for AO obtained using $\mathrm{AO}$ index \\
\hline $\mathrm{NACI}_{\mathrm{NAO}}$ & NACI for NAO obtained using NAO index \\
\hline NAO & North Atlantic Oscillation, the first EOF mode of the winter mean SLP over the North Atlantic $\left(20^{\circ}-80^{\circ} \mathrm{N}, 90 \mathrm{~W}^{\circ}-40^{\circ} \mathrm{E}\right)$ \\
\hline NPCI & $\begin{array}{l}\text { North Pacific center intensity index, the area-averaged SLP anomalies over the North Pacific }\left(25^{\circ}-60^{\circ} \mathrm{N}, 150^{\circ} \mathrm{E}-120^{\circ} \mathrm{W} \text {, }\right. \\
\text { denoted by the red box in Fig. } 5 \mathrm{a}) \text { obtained by regressing winter mean SLP onto a simultaneous index }\end{array}$ \\
\hline $\mathrm{NPCI}_{\mathrm{AO}}$ & NPCI for AO obtained using AO index \\
\hline $\mathrm{NPCI}_{\mathrm{NPM}}$ & NPCI for NPM obtained using NPM index \\
\hline NPM & North Pacific mode, the first EOF mode of the winter mean SLP over the North Pacific $\left(20^{\circ}-65^{\circ} \mathrm{N}, 120^{\circ} \mathrm{E}-120^{\circ} \mathrm{W}\right)$ \\
\hline $\mathrm{PC}$ & Principal component \\
\hline PNA & Pacific-North America \\
\hline$r_{\mathrm{AO}, \mathrm{WNAT}}$ & Correlation coefficient between the AO and WNAT indices \\
\hline$r_{\mathrm{NAO}, \mathrm{NPM}}$ & Correlation coefficient between the NAO and NPM indices \\
\hline$r_{\mathrm{NPM}, \mathrm{WNAT}}$ & Correlation coefficient between the NPM and WNAT indices \\
\hline SAT & Surface air temperature \\
\hline SE1 & The first strong epoch for $\mathrm{NPCI}_{\mathrm{AO}}(1937-59)$ \\
\hline SE2 & The second strong epoch for $\mathrm{NPCI}_{\mathrm{AO}}(1986-2008)$ \\
\hline SLP & Sea level pressure \\
\hline WE & The weak epoch for $\mathrm{NPCI}_{\mathrm{AO}}(1963-85)$ \\
\hline WNAT & $\begin{array}{l}\text { Western North American temperature index, the area-averaged winter mean SAT over western North America } \\
\left(30^{\circ}-60^{\circ} \mathrm{N}, 110^{\circ}-135^{\circ} \mathrm{W} \text {, denoted by the red boxes in Figs. } 4 \mathrm{j}-1\right)\end{array}$ \\
\hline
\end{tabular}

1920 to 2010 . Here, the winter 1920 refers to the $1919 / 20$ winter, and the winter means are calculated by averaging the monthly mean data of December, January, and February. The two-tailed Student's $t$ test is adopted to evaluate the confidence level of linear correlation and regression.

\section{Observed evidence for the time-varying patterns and teleconnections of the AO during the winters of 1920-2010}

\section{a. Pattern and teleconnections of the AO obtained from the winters of 1920-2010}

Figure 1a shows the EOF1 mode of the wintertime SLP anomalies over the extratropical Northern Hemisphere $\left(20^{\circ}-90^{\circ} \mathrm{N}\right)$ derived from the HadSLP2 $\mathrm{r}$ dataset for 1920-2010. The normalized time series of the corresponding PC is shown in Fig. 1d (red line). The EOF1 pattern explains $31.9 \%$ of the total variance and can be clearly separated from the other eigenvalues based on the criterion proposed by North et al. (1982). The EOF1 pattern (Fig. 1a) is characterized by a seesaw in the SLP anomalies between the Arctic and the Northern Hemispheric midlatitudes. An anomalous low SLP center is located in the Arctic region, and two anomalous high SLP centers are observed over the North Pacific and North Atlantic. To check the dependence of the results on datasets, the SLP data from the 20CR-V2c and ERA20C datasets were also analyzed. The EOF1 pattern obtained from 20CR-V2c (ERA-20C) accounts for $29.2 \%(30.9 \%)$ of the total variance, which is comparable to that of HadSLP2r (31.9\%). The wintertime AO pattern in 20CR-V2c and ERA-20C (Figs. 1b,c) both resemble the wintertime $\mathrm{AO}$ pattern derived from HadSLP2r (Fig. 1a), with a spatial correlation coefficient of 0.99 between them. The corresponding PCs from 20CR-V2c and ERA-20C are also highly correlated to the PCs from HadSLP2r, and the temporal correlation coefficients of both exceed 0.98 (Fig. 1d). These results suggest that the wintertime AO variability during 1920-2010 is consistent among the three datasets.

The climate anomaly over the Northern Hemisphere, especially in the SAT, is tightly linked to the AO variability (e.g., Thompson and Wallace 2001). Figure 1e displays the SAT anomalies over the Northern Hemisphere regressed onto the normalized wintertime $\mathrm{AO}$ index in the HadSLP2r dataset. It shows that the positive AO corresponds to significant warming over northern 


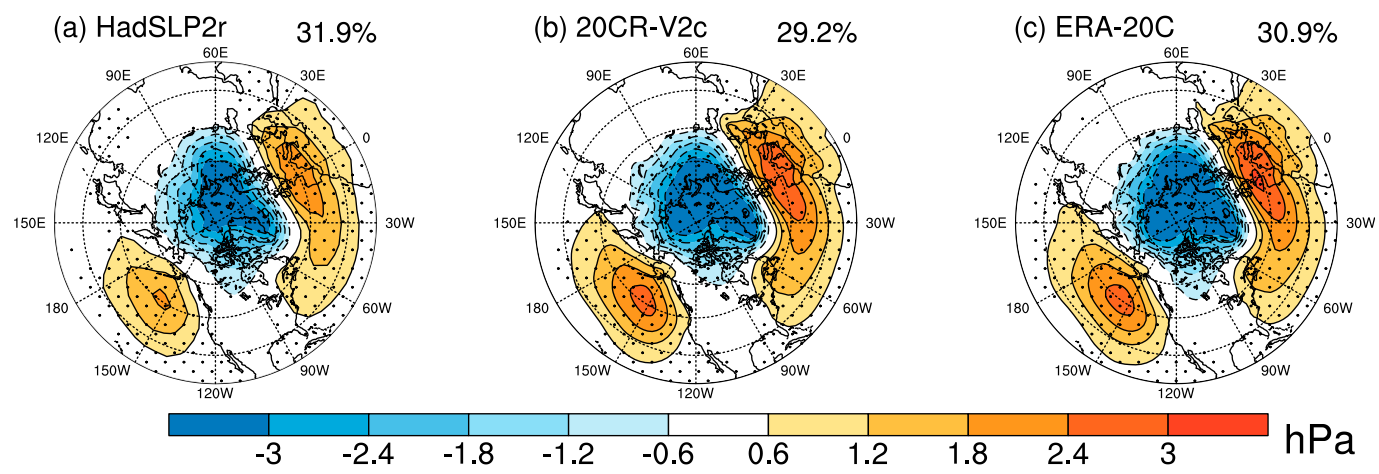

(d) PC1
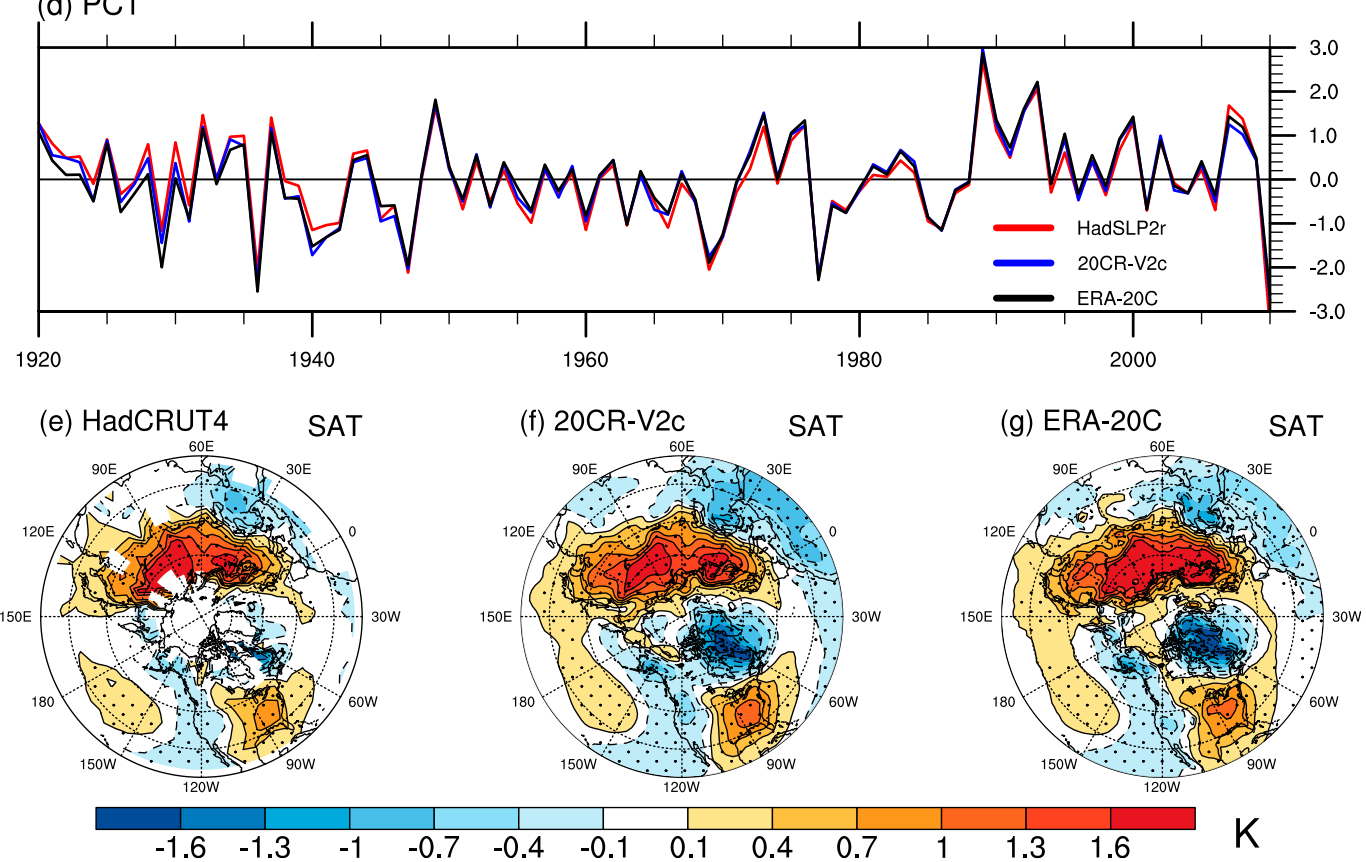

FIG. 1. The winter (December-February) mean AO pattern obtained via regressing the winter mean SLP onto the normalized PC time series of the first EOF mode of the SLP anomalies over the extratropical Northern Hemisphere $\left(20^{\circ}-90^{\circ} \mathrm{N}\right)$ during 1920-2010 in the (a) HadSLP2r, (b) 20CR-V2c, and (c) ERA-20C datasets. (d) The normalized PC time series [i.e., AO indices used in (a)-(c)]. (e)-(g) As in (a)-(c), but for the winter mean SAT [the HadCRUT4 dataset is used in (e)]. Dots in (a)-(c) and (e)-(g) indicate the $99 \%$ and $90 \%$ confidence level based on a two-tailed Student's $t$ test, respectively.

Eurasia and southeastern North America and significant cooling over northern Africa and western and northeastern North America. The above signals are also apparent and consistent in the 20CR-V2c and ERA-20C datasets (Figs. 1f,g). These results suggest that the pattern and teleconnections of the wintertime $\mathrm{AO}$ are consistent between the observations and reanalysis datasets during 1920-2010.

\section{b. Time-varying AO pattern during the winters of 1920-2010}

Previous studies found that the spatial structure of the $\mathrm{AO}$ is not stationary under certain specific conditions
(Honda and Nakamura 2001; Castanheira and Graf 2003; Zhao and Moore 2009; Sun and Tan 2013; Shi and Nakamura 2014; Dai and Tan 2017). In this section, we investigate whether the wintertime AO pattern itself is variable during the different periods of 1920-2010. To clarify the variability of the wintertime AO pattern itself during 1920-2010, we calculate the standard deviation of the sliding AO pattern during 1920-2010 with a 23-yr window (Fig. 2). This is done in two steps. First, we regress the winter mean AO index onto SLP in 23-yr sliding windows during 1920-2010 (i.e., in the winters of 1920-42, 1921-43, ..., 1988-2010). Here, the AO index in each sliding window is extracted from the $\mathrm{AO}$ index in 


\section{Uncertainty of $\mathrm{AO}$ pattern}

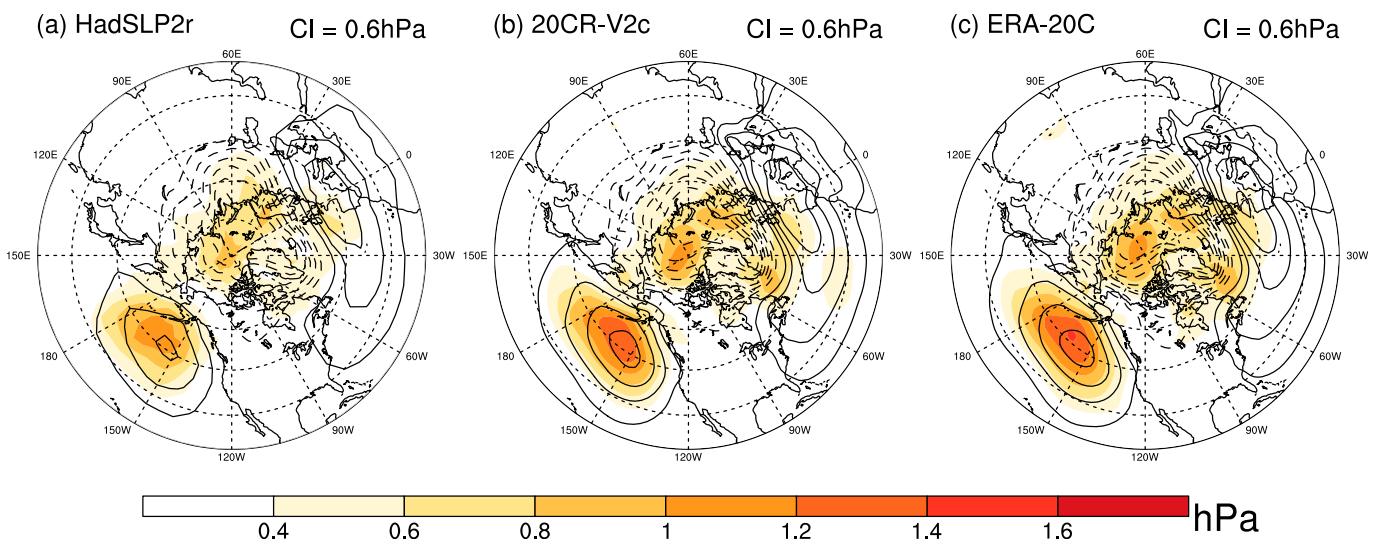

FIG. 2. (a) The AO pattern as in Fig. 1a (contours) overlaid with the standard deviation of the sliding AO pattern with a 23-yr sliding window during the winters of 1920-2010 (shading) based on SLP from the HadSLP2r dataset. See section 3b for details. (b),(c) As in (a), but based on SLP from the 20CR-V2c and ERA-20C datasets, respectively.

Fig. 1d. A total of $69 \mathrm{AO}$ patterns will be obtained in this step. Second, we calculate the standard deviation among these $69 \mathrm{AO}$ patterns to get the standard deviation of the sliding AO pattern. The choice of the $23-\mathrm{yr}$ window is somewhat arbitrary because the window of approximately 20 years is often used to investigate decadal changes (e.g., Wang et al. 2010; Shi and Nakamura 2014). We also tested windows with other widths (e.g., 21,25 , and 31 years), and the main results remain almost unchanged (figures not shown).

The maxima of the standard deviation of the sliding AO patterns are mainly located over the North Pacific in all three datasets (Fig. 2), and they almost overlap with the Pacific center of the AO pattern derived during 1920-2010 (Figs. 1a-c, 2). This suggests that the intensity of the Pacific center of the wintertime AO has large variability during 1920-2010. In contrast, the variability of the Atlantic center in the wintertime AO pattern is much weaker compared with the Pacific center. It suggests that the Atlantic center of the wintertime AO pattern remains relatively invariant during 1920-2010. Although the amplitudes of the variability of the wintertime AO pattern in HadSLP2r (Fig. 2a) are overall weaker than those in 20CR-V2c and ERA-20C (Figs. 2b,c), plausibly because of the low horizontal resolution in HadSLP2r, their patterns resemble each other and are consistent among the three datasets. These results suggest that the Pacific center of the wintertime AO pattern has strong decadal changes during 1920-2010.

To analyze the changes in the intensity of the three AO centers in more detail, the North Pacific center intensity index (NPCI), the North Atlantic center intensity index (NACI), and the Arctic center intensity index (ACI) are defined to represent the intensities of the three centers in the wintertime AO pattern. They are defined as the area-averaged AO-related SLP anomalies (i.e., the SLP anomalies regressed onto the wintertime AO index) over the North Pacific $\left(25^{\circ}-60^{\circ} \mathrm{N}, 150^{\circ} \mathrm{E}-\right.$ $\left.120^{\circ} \mathrm{W}\right)$, the North Atlantic $\left(20^{\circ}-55^{\circ} \mathrm{N}, 80^{\circ} \mathrm{W}-40^{\circ} \mathrm{E}\right)$, and the Arctic $\left(55^{\circ}-90^{\circ} \mathrm{N}, 0^{\circ}-360^{\circ}\right)$ and are denoted as $\mathrm{NPCI}_{\mathrm{AO}}, \mathrm{NACI}_{\mathrm{AO}}$, and $\mathrm{ACI}_{\mathrm{AO}}$, respectively. Figure 3 displays the sliding intensity of the three indices with the 23-yr window during the winters of 1920-2010. The variability of the wintertime AO pattern is weaker in HadSLP2r than in the other two datasets, so the results from HadSLP2r use the right-hand $y$ axis with a smaller scale in Fig. 3 for easy comparison with the other two datasets. It reveals that the $\mathrm{NPCI}_{\mathrm{AO}}$ experiences significant multidecadal fluctuations in all datasets (Fig. 3a). The amplitude of the $\mathrm{NPCI}_{\mathrm{AO}}$ is large before the 1950s, and it decreases rapidly afterward and reaches a minimum around 1974. After that, it increases slowly in the 1990s (Fig. 3a). This feature, which is consistent among the three datasets, confirms that the multidecadal fluctuations of $\mathrm{NPCI}_{\mathrm{AO}}$ are robust during 1920-2010. The minimum of the $\mathrm{NPCI}_{\mathrm{AO}}$ is $0.2(0.5) \mathrm{hPa}$ in the year 1974, which represents the 23-yr window for the winters of 1963-85 in HadSLP2r (20CR-V2c and ERA-20C), and the maxima before and after 1974 are around $1.2(2.0) \mathrm{hPa}$ and $0.9(1.5) \mathrm{hPa}$ in the years 1948 and 1997, which represents the 23-yr window for the winters of 1937-59 and 1986-2008, respectively, in HadSLP2r (20CR-V2c and ERA-20C). Compared with the large variability in the strength of the Pacific center of the AO pattern (i.e., $\mathrm{NPCI}_{\mathrm{AO}}$ ), the strength of the North Atlantic and Arctic centers of the AO pattern 

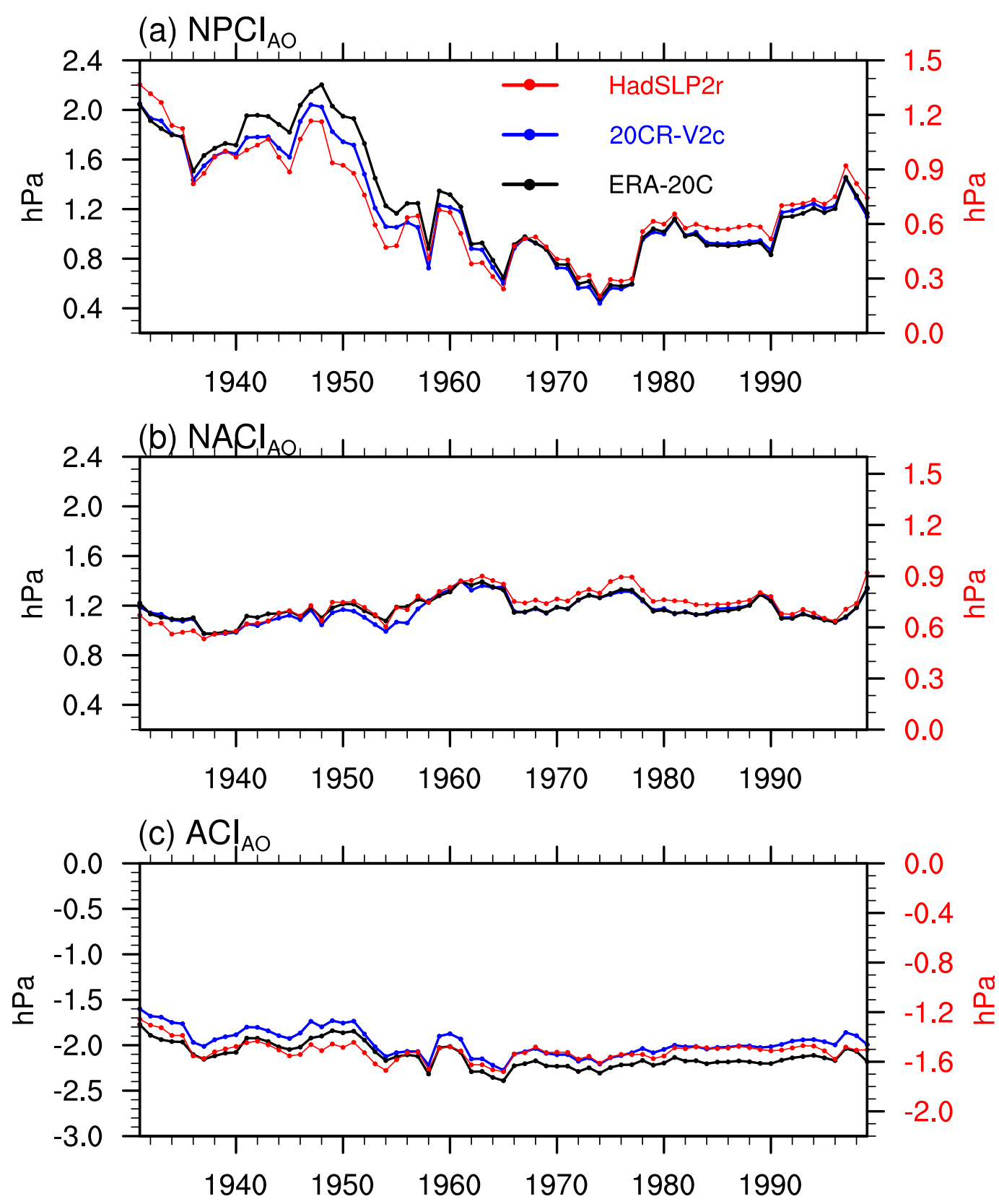

FIG. 3. (a) Sliding $\mathrm{NPCI}_{\mathrm{AO}}$ (hPa) in a 23-yr sliding window during the winters of 1920-2010 in the 20CR-V2c and ERA-20C datasets (using left $y$ axis) and the HadSLP2r dataset (using right $y$ axis). See section $3 b$ and Table 1 for details. (b),(c) As in (a), but for the $\mathrm{NACI}_{\mathrm{AO}}$ and $\mathrm{ACI}_{\mathrm{AO}}$, respectively.

(i.e., $\mathrm{NACI}_{\mathrm{AO}}$ and $\mathrm{ACI}_{\mathrm{AO}}$ ) remains relatively invariant (Figs. 3b,c). The $\mathrm{NACI}_{\mathrm{AO}}$ and $\mathrm{ACI}_{\mathrm{AO}}$ are always around $0.7(1.2) \mathrm{hPa}$ and $-1.7(-2.0) \mathrm{hPa}$, respectively, in HadSLP2r (20CR-V2c and ERA-20C) during the winters of 1920-2010. These results further confirm that the Pacific center of the AO experiences significant multidecadal changes, whereas the Atlantic and Arctic centers of the AO remain almost unchanged during the winters of 1920-2010. c. Time-varying AO teleconnections during the winters of 1920-2010

Considering the large variability of the Pacific center in the AO pattern, the climate anomalies associated with the AO may also experience significant differences during the winters of 1920-2010. To investigate the possibility of changes in circulation and climate anomalies associated with the variability in the AO pattern, 
three typical 23-yr periods with the smallest and largest $\mathrm{NPCI}_{\mathrm{AO}}$ are singled out. One spans the winters of 196385 , corresponding to the minimum of sliding $\mathrm{NPCI}_{\mathrm{AO}}$ in 1974 (Fig. 3a), and the other two span the winters of 1937-59 and 1986-2008, corresponding to the maximum of sliding $\mathrm{NPCI}_{\mathrm{AO}}$ in 1948 and 1997 before and after 1974 (Fig. 3a). For convenience, these three periods are marked as weak epoch (WE, 1963-85), strong epoch 1 (SE1, 1937-59), and strong epoch 2 (SE2, 1986-2008), respectively. Because the results among the different datasets are highly consistent (figures not shown), only the results based on the 20CR-V2c reanalysis dataset are shown in the following sections.

Figure 4 displays the anomalies of SLP, 500-hPa geopotential height, 700-hPa winds, and SAT regressed onto the winter mean $\mathrm{AO}$ index in the three epochs. Significant positive SLP anomalies are observed over the North Pacific in SE1 and SE2 (Figs. 4a,c), although the intensity of the SLP anomalies is somewhat weaker in SE2 than those in the SE1 (Fig. 4c). In contrast, the positive SLP anomalies over the North Pacific almost disappear in WE (Fig. 4b). Similar differences can be seen in the 500-hPa geopotential height field (Figs. 4df), which shows significant high pressure center over the North Pacific in SE1 and SE2 (Figs. 4d,f), but not in WE (Fig. 4e). The high pressure center over the North Pacific is equivalently barotropic and can also be seen in the 700-hPa wind field (Figs. 4g-i). A pronounced anticyclonic anomaly is observed in the lower troposphere over the North Pacific in SE1 and SE2 (Figs. 4g,i). The northerly anomalies of this anticyclone over western North America are favorable for advection of cold air from the high latitudes to the western coast of North America, thereby inducing cold anomalies over western North America (Figs. 4j,1). In contrast, the anticyclonic anomaly over the North Pacific is weak and almost invisible in WE (Fig. 4h). As a result, the cold advection and SAT anomalies over western North America are both weak in WE (Fig. 4k). These results suggest that accompanied with the time-varying amplitude of the Pacific center of AO pattern, the influences of the AO on the SAT over the Pacific-North American region also experience multidecadal changes.

\section{Revisiting the nature of the $\mathrm{AO}$ from the perspective of decadal change}

The results in section 3 indicate that the winter mean AO pattern defined as the leading EOF of SLP anomalies over the extratropical Northern Hemisphere is nonstationary and that its Pacific center experiences clear multidecadal fluctuations during 1920-2010. The resultant AO teleconnection (i.e., the relationship between $\mathrm{AO}$ and the western North American SAT) also displays distinct differences between SE1/SE2 and WE (Fig. 4). In SE1, the AO-related climate anomalies over the Pacific-North American region quite resemble those related to the North Pacific variability (e.g., Johnson and Feldstein 2010; Franzke et al. 2011; Yuan et al. 2015). To confirm these similarities, several variables were regressed onto the NPM index during the winters of 1920-2010 to display the patterns associated with the dominant regional variability over the North Pacific (Fig. 5). It shows that the positive phase of the NPM index is featured with pronounced quasibarotropic high pressure anomalies extending from surface to the middle troposphere over the North Pacific (Figs. 5a-c), which are quite similar to those related to the AO in SE1 and SE2 (Figs. 4a,c,d,f,g,i). The 500-hPa geopotential height field shows a typical PNA pattern (Fig. 5b), indicating that the NPM is almost equivalent to the PNA pattern (see discussions in section 2). Significant cold anomalies are observed over northwestern North America (Fig. 5d), likely attributed to cold advection by the northerly anomalies there (Fig. 5c).

On one hand, the high similarity between AO-related climate anomalies in SE1/SE2 (left and right columns in Fig. 4) and NPM-related climate anomalies (Fig. 5) is consistent with Quadrelli and Wallace (2004). On the other hand, this high similarity raises a question: Which is the fundamental mode of the climate variability over the North Pacific, the NPM or the AO? In some sense, the answer to this question is related to the physical nature of the $\mathrm{AO}$ especially regarding the Pacific center of the AO discussed by many previous studies (e.g., Deser 2000; Wallace 2000; Ambaum et al. 2001; Rogers and McHugh 2002; Wallace and Thompson 2002; Feldstein and Franzke 2006; Itoh 2008). In this section, we will try to address this question from the perspective of decadal changes.

\section{a. The NPM as the fundamental mode over the North Pacific}

To answer the question whether the NPM or the AO is the fundamental mode of climate variability over the North Pacific, the possible multidecadal changes of the NPM pattern are examined during the winters of 19202010. Figure 6 shows the intensity of the NPM-related Pacific center in a 23-yr sliding window, marked as $\mathrm{NPCI}_{\mathrm{NPM}}$. Similar to the NPCI $\mathrm{I}_{\mathrm{AO}}$ used in section $3 \mathrm{~b}$, the $\mathrm{NPCI}_{\mathrm{NPM}}$ is defined as the area-averaged NPM-related SLP anomalies over the North Pacific $\left(25^{\circ}-60^{\circ} \mathrm{N}, 150^{\circ} \mathrm{E}-\right.$ $\left.120^{\circ} \mathrm{W}\right)$. It reveals that unlike the time-varying $\mathrm{NPCI}_{\mathrm{AO}}$, the $\mathrm{NPCI}_{\mathrm{NPM}}$ remains almost the same (approximately $2.3 \mathrm{hPa}$ ) during the entire 1920-2010 period (Fig. 6). Therefore, the Pacific center associated with the NPM 


\section{AO-regressed variables}

\section{SE1(1937-1959)}

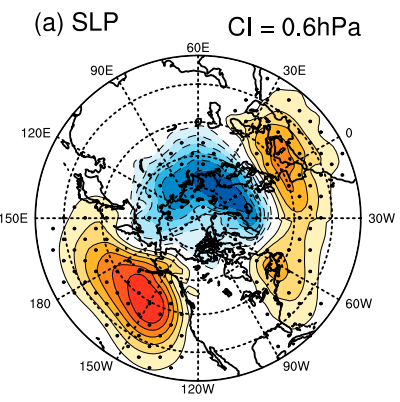

(d) Z500

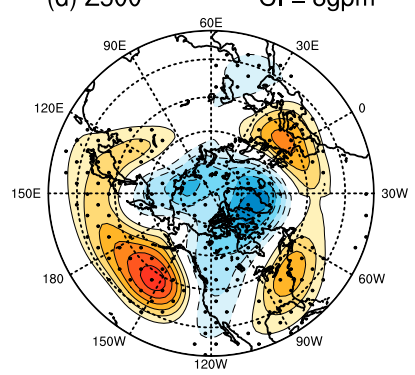

(g) UV700
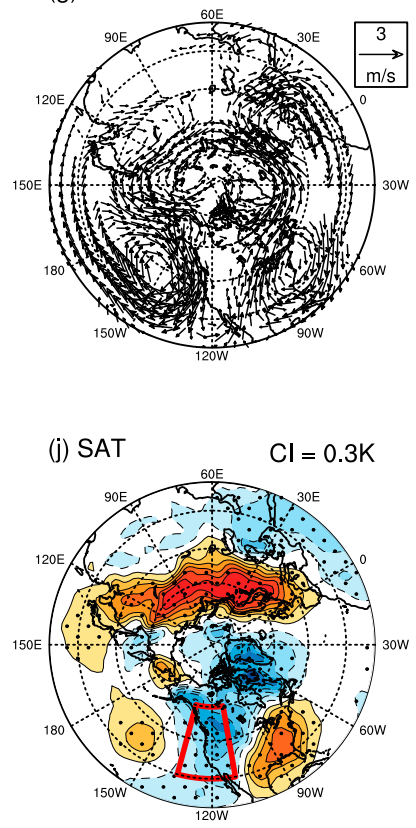

WE(1963-1985)
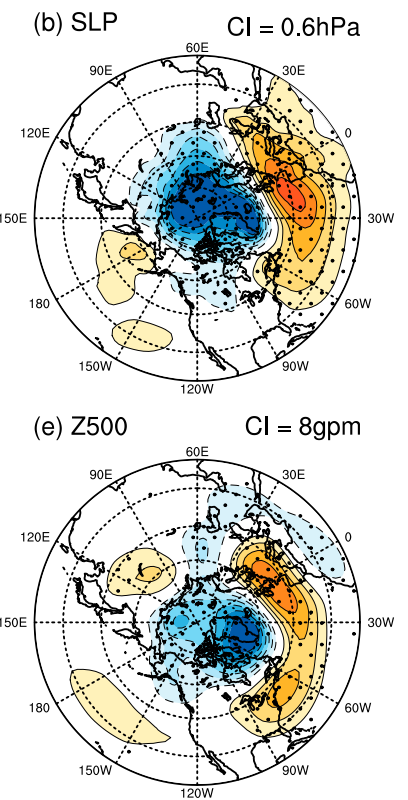

(h) UV700
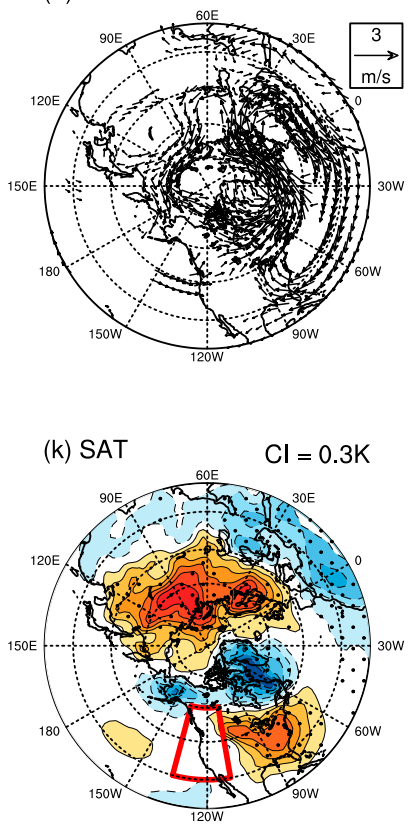

SE2(1986-2008)
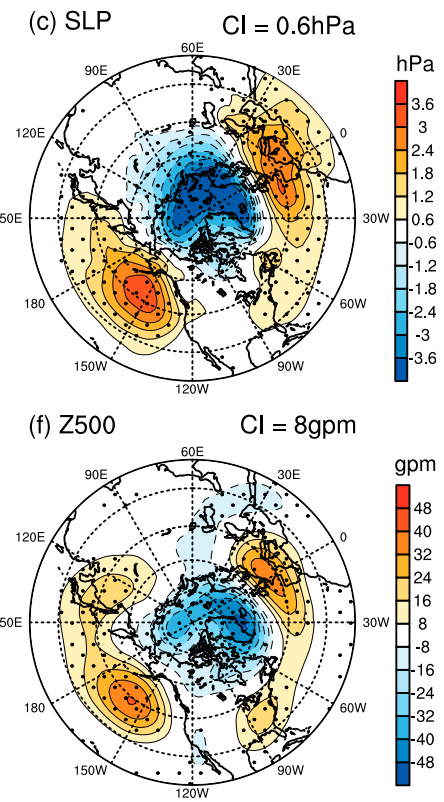

(i) UV700
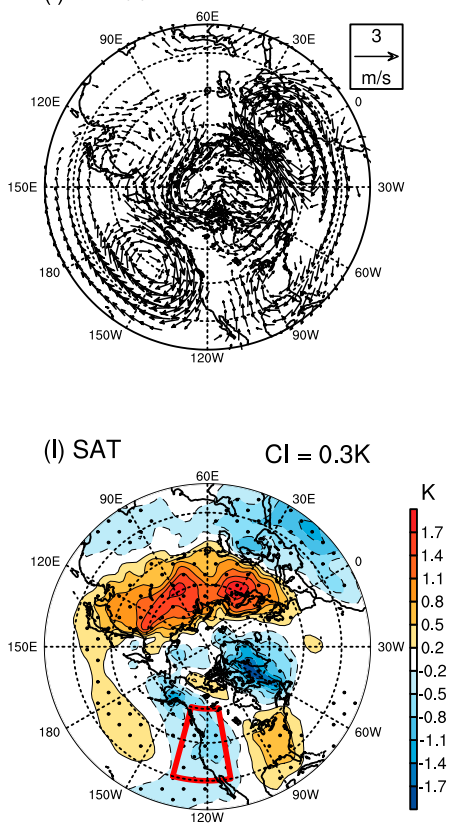

FIG. 4. The winter mean SLP regressed on the simultaneous normalized AO index during (a) SE1 (1937-59), (b) WE (1963-85), and (c) SE2 (1986-2008) in the 20CR-V2c dataset. (d)-(f), (g)-(i), and (j)-(l) As in (a)-(c), but for 500-hPa geopotential height field, 700-hPa winds, and SAT, respectively. Shading intervals are $0.6 \mathrm{hPa}$ in (a)-(c), $8 \mathrm{gpm}$ in (d)-(f), and $0.3 \mathrm{~K}$ in (j)-(l). Dots in (a)-(f) and (j)-(l) indicate the $90 \%$ confidence level based on a twotailed Student's $t$ test. Vectors exceeding the $90 \%$ confidence level are shown in (g)-(i). Red boxes in (j)-(l) indicate the region to define WNAT. 


\section{NPM-regressed variables}

(a) SLP

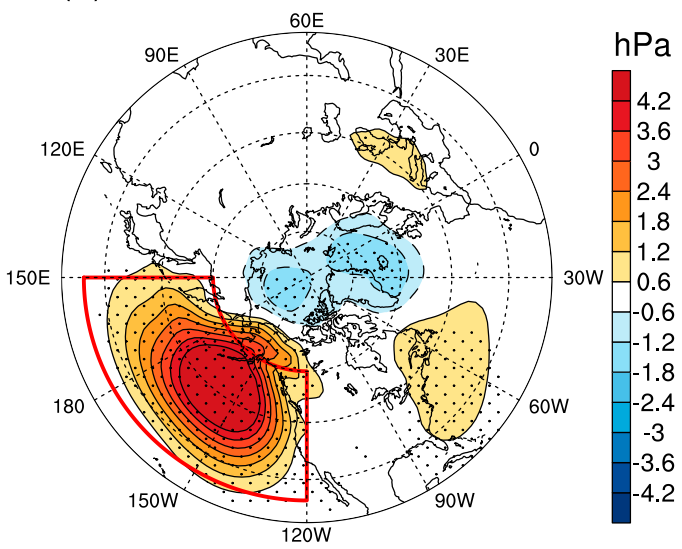

(c) UV700

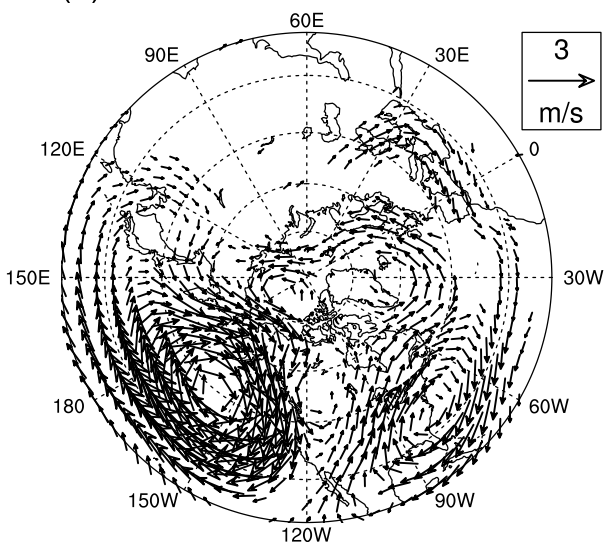

(b) Z500

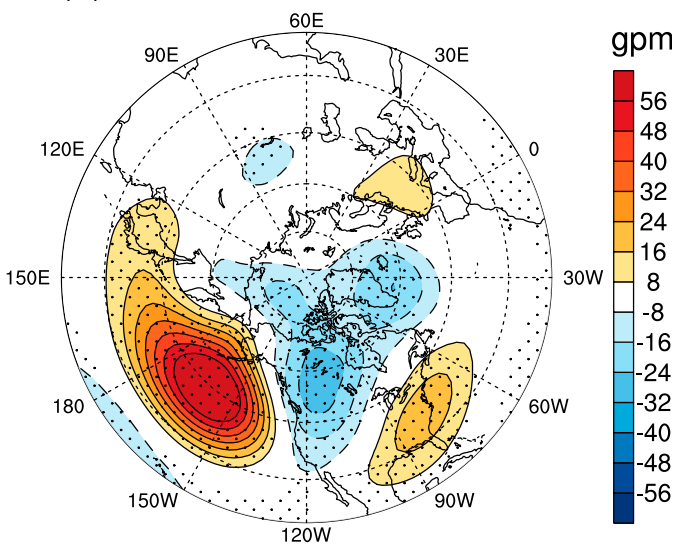

(d) SAT

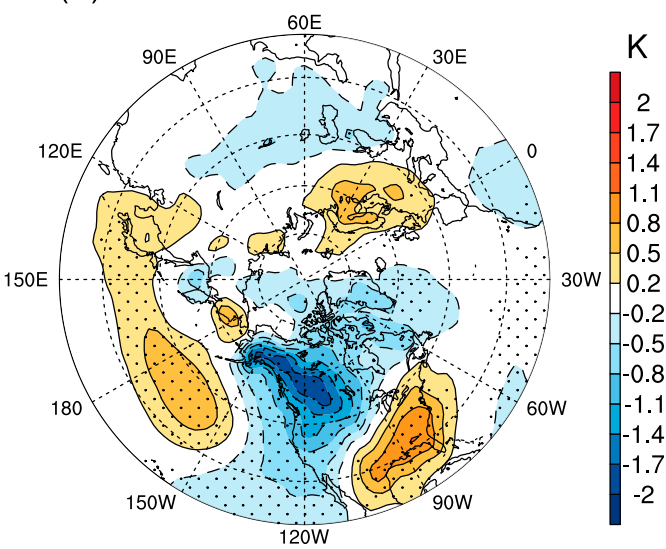

FIG. 5. (a) The winter mean SLP regressed on the simultaneous normalized NPM index during 1920-2010 in the 20CR-V2c dataset. (b)-(d) As in (a), but for 500-hPa geopotential height field, 700-hPa winds, and SAT, respectively. Dots in (a), (b), and (d) indicate the $99 \%$ confidence level based on a two-tailed Student's $t$ test. Vectors exceeding the $99 \%$ confidence level are shown in (c). The red box in (a) indicates the region to define NPCI.

pattern is stationary and does not have decadal changes. To further examine the possible decadal changes in the NPM-related climate anomalies over western North America, we define an index as the area-averaged winter mean SAT over western North America $\left(30^{\circ}-60^{\circ} \mathrm{N}\right.$, $\left.110^{\circ}-135^{\circ} \mathrm{W}\right)$, denoted as the WNAT index. The sliding correlation coefficient between the winter mean NPM index and WNAT index (marked as $r_{\mathrm{NPM}, \mathrm{WNAT}}$ ) remains around -0.8 during the entire $1920-2010$ period. This result suggests that the SAT variability over western North America is always closely tied to the NPM during 1920-2010. This stable NPM-WNAT relation can be attributed to the invariant Pacific center associated with the NPM pattern (Fig. 6). Compared with the time-varying intensity of the AO-related Pacific center (Fig. 3a), the relatively invariant intensity of the
NPM-related Pacific center (Fig. 6) suggests that the NPM rather than the AO is more likely an inherent and fundamental mode of the atmosphere variability over the North Pacific in boreal winter.

\section{$b$. The stable similarity between the $A O$ and $N A O$}

It was generally accepted that the wintertime AO and NAO are quite similar and somewhat difficult to distinguish during a specified period (e.g., Deser 2000; Rogers and McHugh 2002; Feldstein and Franzke 2006). Here we will check whether the similarity between AO and NAO has decadal changes during the winters of 1920-2010. Figure 7a shows the wintertime SLP pattern of NAO obtained by regressing the winter mean NAO index onto SLP over the extratropical Northern Hemisphere for the winters of 1920-2010. The NAO pattern, 


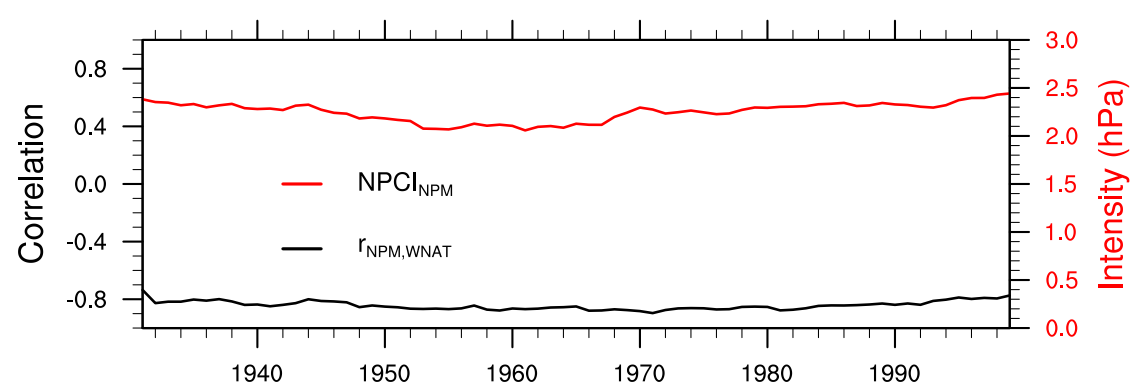

FIG. 6. Sliding correlation coefficients between the winter mean NPM index and WNAT index (i.e., $r_{\mathrm{NPM}, \mathrm{WNAT}}$ ) with a 23-yr sliding window during the winters of 1920-2010 (black line) and the sliding North Pacific center intensity index of the NPM pattern (i.e., $\mathrm{NPCI}_{\mathrm{NPM}}$ ) with a 23-yr sliding window during the winters of 1920-2010 (red line). Data are from the 20CR-V2c dataset.

especially the dipole structure over the North Atlantic, closely resembles the AO pattern, and the Pacific center of the NAO is weaker than that in the AO pattern (Fig. 1b). The pattern correlation coefficient between the SLP patterns of AO and NAO is 0.96 , implying that the wintertime $\mathrm{AO}$ and NAO may always remain similar during different epochs of 1920-2010.

To verify the above hypothesis, the sliding correlation coefficients with a 23-yr sliding window were calculated between the wintertime NAO and AO (Fig. 7b). It reveals that the temporal correlation coefficients between the NAO and AO indices almost always exceed 0.9 and that the pattern correlation coefficients between the sliding SLP patterns of AO and NAO over the extratropical Northern Hemisphere $\left(20^{\circ}-90^{\circ} \mathrm{N}, 0^{\circ}-360^{\circ}\right)$ almost always exceed 0.95 during the entire 1920-2010 period (Fig. 7b). This result confirms that the wintertime $\mathrm{AO}$ and NAO are indeed difficult to distinguish during 1920-2010, supporting previous studies (e.g., Rogers and McHugh 2002; Feldstein and Franzke 2006). In addition, the intensity of the Atlantic center of the NAO, which is defined as the area-averaged NAO-related SLP anomalies over the North Atlantic $\left(20^{\circ}-55^{\circ} \mathrm{N}, 80^{\circ} \mathrm{W}-40^{\circ} \mathrm{E}\right)$ and marked as $\mathrm{NACI}_{\mathrm{NAO}}$, remains almost unchanged during the entire 1920-2010 period (Fig. 7b). This result suggests that the NAO is very likely the inherent and fundamental mode of atmosphere variability over the North Atlantic in boreal winter.

Wallace (2000) suggested that the NAO and AO are different names for the same variability, not different patterns of variability. He also proposed that the difference between NAO and AO lies in whether the North Atlantic variability is interpreted as a regional pattern controlled by processes within the North Atlantic sector or as an annular mode whose strongest teleconnections are located over the North Atlantic sector. On one hand, our results presented in sections $4 \mathrm{a}$ and $4 \mathrm{~b}$ confirm that the NPM and NAO are the inherent and fundamental regional modes over the North Pacific and North Atlantic regions in view of their stationary pattern over the two regions, respectively. On the other hand, the time-invariant consistency between the AO and NAO (Fig. 7b) and the time-varying North Pacific center of the AO indicate that the AO seems to be fundamentally rooted in the variability not over the North Pacific, but over the North Atlantic. Moreover, the time-varying North Pacific center of the AO implies that the annular structure of the AO very likely arises from the coupling of the atmospheric modes between the North Pacific and North Atlantic.

\section{c. The time-varying Pacific center of the $A O$ and the North Pacific-North Atlantic coupling}

To test the possible linkage between the annular structure of the AO and the coupling of the atmospheric modes between the North Pacific and North Atlantic, the correlation coefficient between the winter mean NAO and NPM indices ( $\left.r_{\mathrm{NAO}, \mathrm{NPM}}\right)$ was employed to represent the strength of coupling between the North Pacific and North Atlantic (Gong et al. 2017). The correlation was calculated in a 23-yr sliding window to show its possible decadal change. It reveals that the temporal variations of the sliding $r_{\text {NAO,NPM }}$ are highly consistent with that of the intensity of the Pacific center of the AO as indicated by $\mathrm{NPCI}_{\mathrm{AO}}$ (Fig. 8a). This result suggests that the Pacific center of the AO is strong (weak) when the NAO is closely (loosely) coupled to the NPM, and vice versa. Figure $8 \mathrm{~b}$ further shows the scatter diagram between $r_{\mathrm{NAO}, \mathrm{NPM}}\left(x\right.$ axis) and $\mathrm{NPCI}_{\mathrm{AO}}(y$ axis $)$, where each dot represents the value in each 23-yr sliding window. The linearly clustered dots and the high correlation coefficient between $r_{\mathrm{NAO}, \mathrm{NPM}}$ and $\mathrm{NPCI}_{\mathrm{AO}}(r=0.9)$ suggest that approximately $80 \%$ of the variance of the intensity of the Pacific center of the $\mathrm{AO}$ (i.e., $\mathrm{NPCI}_{\mathrm{AO}}$ ) is linked to the coupling between the leading regional 
(a) NAO-regressed SLP
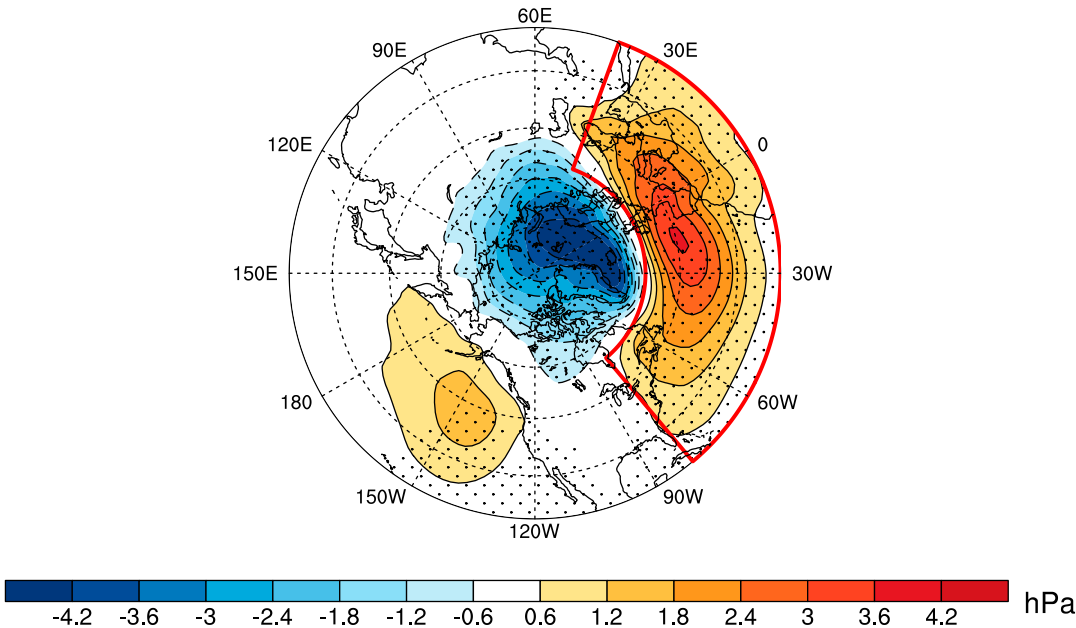

(b)

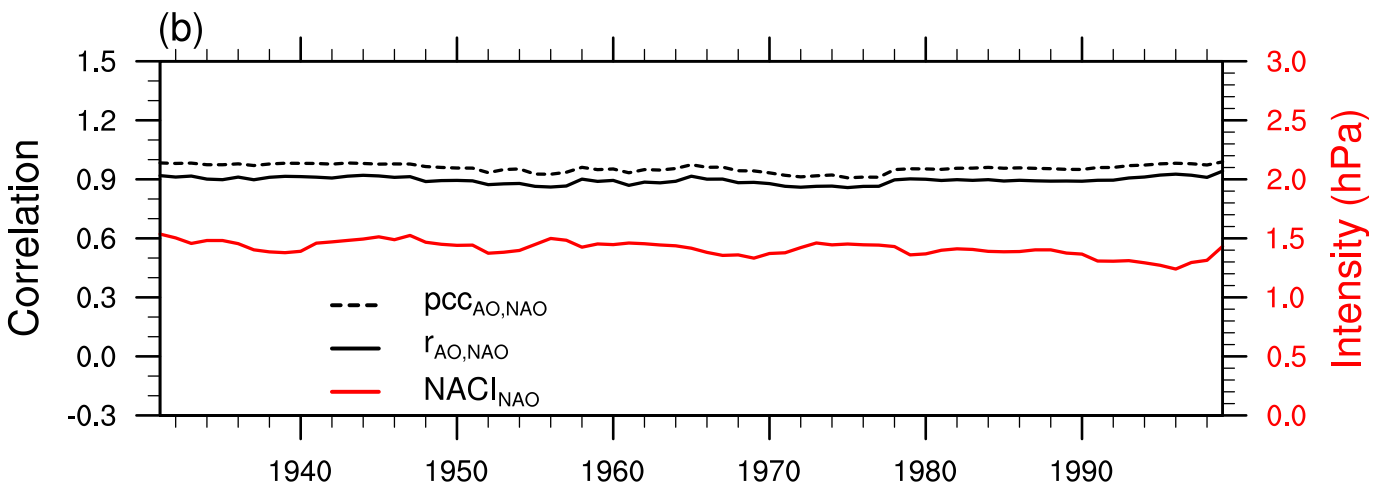

FIG. 7. (a) The winter mean SLP regressed on the simultaneous normalized NAO index during 1920-2010. (b) Sliding temporal correlation coefficient between the winter mean AO and NAO indices (i.e., $r_{\mathrm{AO}, \mathrm{NAO}}$ ) with a 23-yr sliding window (black solid line), sliding pattern correlation coefficient between the SLP patterns of AO and NAO (i.e., pcc $\left.\mathrm{AO}_{\mathrm{NAO}}\right)$ over the extratropical Northern Hemisphere $\left(20^{\circ}-90^{\circ} \mathrm{N}, 0^{\circ}-360^{\circ}\right)$ with a 23 -yr sliding window (black dot line), and the sliding North Atlantic center intensity index of the NAO pattern (i.e., NACI NAO) with a 23-yr sliding window during the winters of 1920-2010. Dots in (a) indicate the $99 \%$ confidence level based on a two-tailed Student's $t$ test. Data are from 20CR-V2c dataset. The red box in (a) indicates the region to define NACI.

modes over the North Atlantic and North Pacific (i.e., between NAO and NPM).

Section $3 \mathrm{c}$ shows that the relationship between the $\mathrm{AO}$ and the winter mean SAT over western North America, which can be measured by the correlation coefficient between the winter mean AO index and the WNAT index (i.e., $r_{\mathrm{AO} \text {,WNAT }}$ ), changes with the intensity of the Pacific center of the AO (i.e., $\mathrm{NPCI}_{\mathrm{AO}}$ ). Here we further examine whether $r_{\mathrm{AO} \text {,WNAT }}$ is linked to the coupling between NAO and NPM. Figure 8a shows the sliding $r_{\mathrm{AO} \text {,WNAT }}$ with a 23 -yr window, where the sign of $r_{\mathrm{AO} \text {,WNAT }}$ is multiplied by -1 for easy comparison with other indices. Clear multidecadal change is observed in the sliding $r_{\text {AO,WNAT }}$ during 1920-2010, and its temporal evolution is highly consistent with that of $\mathrm{NPCI}_{\mathrm{AO}}$ and $r_{\mathrm{NAO}, \mathrm{NPM}}$. The scatter diagrams between
$r_{\mathrm{AO}, \mathrm{WNAT}}\left(y\right.$ axis) and $\mathrm{NPCI}_{\mathrm{AO}}(x$ axis) (Fig. $8 \mathrm{c})$ and between $r_{\text {AO,WNAT }}\left(y\right.$ axis) and $r_{\mathrm{NAO}, \mathrm{NPM}}(x$ axis) (Fig. 8d) confirm the results in Fig. 8a. The linearly clustered dots and the high correlation coefficients between $r_{\mathrm{AO}, \mathrm{WNAT}}$ and $\mathrm{NPCI}_{\mathrm{AO}}(r=0.88)$ and between $r_{\mathrm{AO}, \mathrm{WNAT}}$ and $r_{\mathrm{NAO}, \mathrm{NPM}}(r=0.87)$ confirm that the relationship between the AO and the winter mean SAT over western North America also depends on the intensity of the Pacific center of the AO and the coupling between NAO and NPM.

\section{Summary}

In this study, the multidecadal fluctuations of the pattern and teleconnections of the $\mathrm{AO}$ are investigated during the winters of 1920-2010 based on observation 
(a)

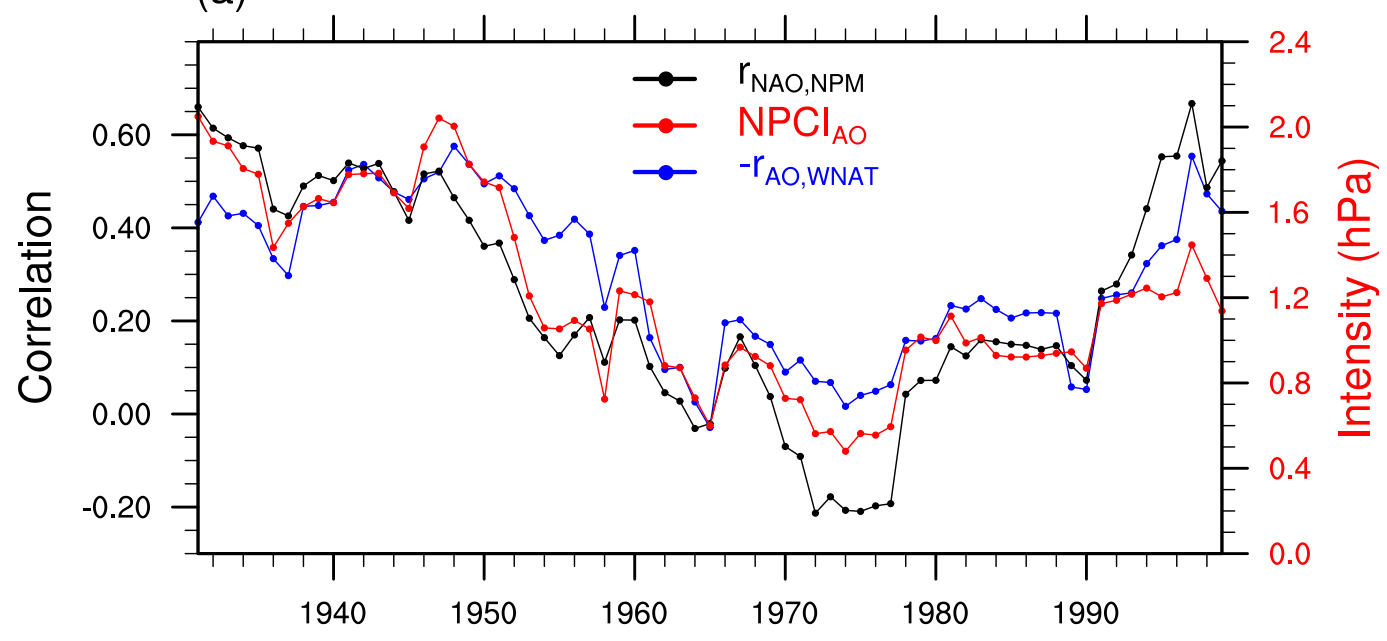

(b) $r=0.9$

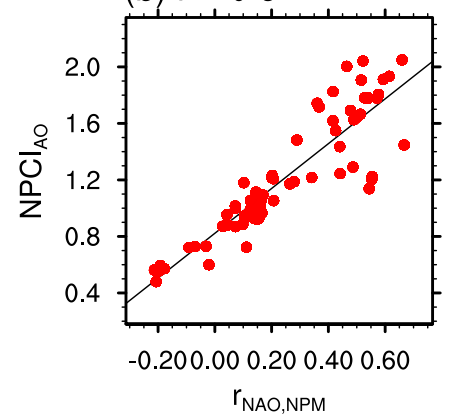

(c) $r=0.88$

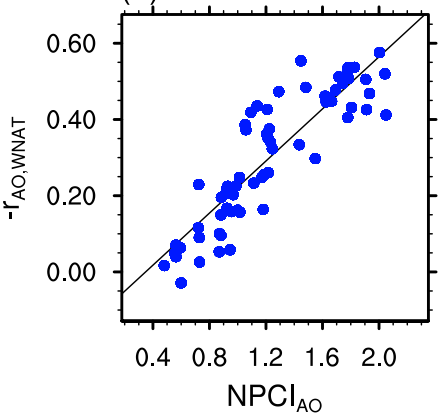

(d) $r=0.87$

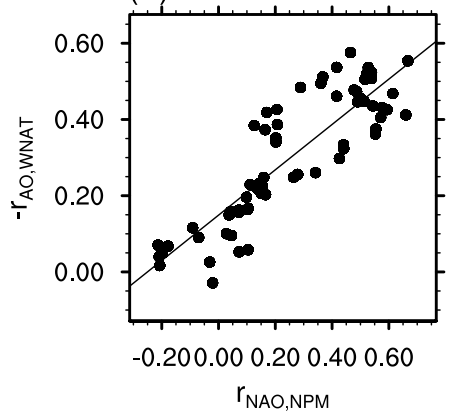

FIG. 8. (a) Sliding correlation coefficient between the winter mean AO and negative WNAT indices (i.e., $-r_{\mathrm{AO}}$ WNAT) with a 23-yr window (blue line, using left $y$ axis), sliding correlation coefficient between the winter mean NAO and NPM indices (i.e., $r_{\mathrm{NAO}, \mathrm{NPM}}$ ) with a 23 -yr window (black line, using left $y$ axis), and sliding $\mathrm{NPCI}_{\mathrm{AO}}(\mathrm{hPa})$ in a 23-yr sliding window (red line, using right $y$ axis). (b) Scatterplot of sliding $r_{\mathrm{NAO}, \mathrm{NPM}}\left(x\right.$ axis) vs sliding $\mathrm{NPCI}_{\mathrm{AO}}$ ( $y$ axis). (c),(d) As in (b), but for the sliding $\mathrm{NPCI}_{\mathrm{AO}}\left(x\right.$ axis) vs the sliding negative $r_{\mathrm{AO}, \mathrm{WNAT}}(y$ axis) and the sliding $r_{\mathrm{NAO}, \mathrm{NPM}}\left(x\right.$ axis) vs the sliding negative $r_{\mathrm{AO}, \mathrm{WNAT}}(y$ axis), respectively. Data are from the 20CR-V2c dataset.

(HadSLP2r, HadCRU4) and reanalysis (20CR-V2c and ERA-20C) datasets. The intensity of the Pacific center of the wintertime $\mathrm{AO}$ pattern $\left(\mathrm{NPCI}_{\mathrm{AO}}\right)$ in a $23-\mathrm{yr}$ sliding window is shown to experience clear multidecadal changes. The $\mathrm{NPCI}_{\mathrm{AO}}$ is strong before the 1950s and weak afterward, reaching a minimum around 1974, and it recovers slowly in the 1990s. In contrast, the Atlantic center of the wintertime $\mathrm{AO}$ pattern $\left(\mathrm{NACI}_{\mathrm{AO}}\right)$ remains almost unchanged during 1920-2010. Accompanied with the fluctuations of $\mathrm{NPCI}_{\mathrm{AO}}$, the relationship between the AO and the SAT over western North America $\left(r_{\mathrm{AO}, \mathrm{WNAT}}\right)$ also changes. During epochs with a strong Pacific center of the AO, a pronounced anticyclonic center is observed over the North Pacific in the positive phases of AO. It facilitates strong cold advection over the western coast of North America, resulting in significant cold anomalies over western North
America. In contrast, during epochs with a weak Pacific center of the AO, the influences of the AO on the SAT over western North America are weak.

The time-varying Pacific center of the AO motivated a revisit to the nature of the $\mathrm{AO}$ from the perspective of decadal change. On one hand, it was revealed that the North Pacific center in the NPM pattern, the regional dominant atmospheric variability over the North Pacific, and the North Atlantic center in the NAO pattern, the regional dominant atmospheric variability over the North Atlantic, both remain invariant during 1920-2010, suggesting that the NPM and NAO are inherent and fundamental modes of the wintertime climate variability over the North Pacific and North Atlantic, respectively. On the other hand, it was revealed that the AO-related circulation patterns surrounding the North Pacific quite resemble those related to the NPM during SE1 and SE2 
when the strong Pacific center of the AO is observed, but this similarity disappears during WE when the weak Pacific center of the AO is observed. In contrast, the AOrelated circulation patterns surrounding the North Atlantic always resemble those related to the NAO. Moreover, it was found that the intensity of the Pacific center of the AO evolves almost in phase with the strength of coupling between the NPM and NAO. The Pacific center of the AO is strong (weak) when the NAO is closely (loosely) coupled to the NPM, and vice versa. These results suggest that the AO seems to be fundamentally rooted in the variability over the North Atlantic, a finding aligned with those of Ambaum et al. (2001) and Itoh (2008), and that the annular structure of the AO very likely arises from the coupling of the atmospheric modes between the North Pacific and North Atlantic.

The time-varying pattern of the AO might challenge the reliability and validity of using the AO to represent the leading climate variability over the extratropical Northern Hemisphere. Meanwhile, it might also provide a new criterion to test the proposed theories of the AO. Note that the results reported in this study are based on statistics of winter mean variables without examining associated dynamical processes. These phenomena need to be explained by some diagnostic and theoretical studies in the future. Last but not least, it is important to understand why the NPM-NAO coupling shows multidecadal fluctuations. A preliminary inspection suggests that it may be related to the phase of the Atlantic multidecadal oscillation (not shown), and this will be investigated in our future studies.

Acknowledgments. We thank the Editor Mathew Barlow and three anonymous reviewers for their insightful comments that led to improvements to the manuscript. This work was supported by the National Natural Science Foundation of China (41422501, 41721004, and 41605060), the Key Research Program of Frontier Sciences of the Chinese Academy of Sciences (QYZDY-SSW-DQC024), and the Fundamental Research Funds for the Central Universities.

\section{REFERENCES}

Allan, R. J., and T. J. Ansell, 2006: A new globally complete monthly historical gridded mean sea level pressure dataset (HadSLP2): 1850-2004. J. Climate, 19, 5816-5842, https:// doi.org/10.1175/JCLI3937.1.

Ambaum, M. H. P., B. J. Hoskins, and D. B. Stephenson, 2001: Arctic Oscillation or North Atlantic Oscillation? J. Climate 14, 3495-3507, https://doi.org/10.1175/1520-0442(2001)014<3495: $\mathrm{AOONAO}>2.0 . \mathrm{CO} ; 2$.

Castanheira, J. M., and H. F. Graf, 2003: North Pacific-North Atlantic relationships under stratospheric control? J. Geophys. Res., 108, 4036, https://doi.org/10.1029/2002JD002754.
Chen, W., and L. Kang, 2006: Linkage between the Arctic Oscillation and winter climate over East Asia on the interannual timescale: Roles of quasistationary planetary waves. Chin. J. Atmos. Sci., 30, 863-870.

, X. Lan, L. Wang, and Y. Ma, 2013: The combined effects of the ENSO and the Arctic Oscillation on the winter climate anomalies in East Asia. Chin. Sci. Bull., 58, 1355-1362, https:// doi.org/10.1007/s11434-012-5654-5.

Christiansen, B., 2002: On the physical nature of the Arctic Oscillation. Geophys. Res. Lett., 29, 1805, https://doi.org/10.1029/ 2002GL015208.

Compo, G. P., and Coauthors, 2011: The Twentieth Century Reanalysis Project. Quart. J. Roy. Meteor. Soc., 137, 1-28, https:// doi.org/10.1002/qj.776.

Dai, P., and B. Tan, 2017: The nature of the Arctic Oscillation and diversity of the extreme surface weather anomalies it generates. J. Climate, 30, 5563-5584, https://doi.org/10.1175/ JCLI-D-16-0467.1.

Deser, C., 2000: On the teleconnectivity of the "Arctic Oscillation." Geophys. Res. Lett., 27, 779-782, https://doi.org/10.1029/ 1999GL010945.

Feldstein, S. B., and C. Franzke, 2006: Are the North Atlantic Oscillation and the Northern Annular Mode distinguishable? J. Atmos. Sci., 63, 2915-2930, https://doi.org/10.1175/ JAS3798.1.

Franzke, C., S. B. Feldstein, and S. Lee, 2011: Synoptic analysis of the Pacific-North American teleconnection pattern. Quart. J. Roy. Meteor. Soc., 137, 329-346, https://doi.org/10.1002/qj.768.

Giese, B. S., H. F. Seidel, G. P. Compo, and P. D. Sardeshmukh, 2016: An ensemble of ocean reanalyses for 1815-2013 with sparse observational input. J. Geophys. Res. Oceans, 121, 6891-6910, https://doi.org/10.1002/2016JC012079.

Gong, D. Y., S. W. Wang, and J. H. Zhu, 2001: East Asian winter monsoon and Arctic Oscillation. Geophys. Res. Lett., 28, 2073-2076, https://doi.org/10.1029/2000GL012311.

Gong, H., L. Wang, W. Chen, X. Chen, and D. Nath, 2017: Biases of the wintertime Arctic Oscillation in CMIP5 models. Environ. Res. Lett., 12, 014001, https://doi.org/10.1088/1748-9326/ 12/1/014001.

Honda, M., and H. Nakamura, 2001: Interannual seesaw between the Aleutian and Icelandic lows. Part II: Its significance in the interannual variability over the wintertime Northern Hemisphere. J. Climate, 14, 4512-4529, https://doi.org/ 10.1175/1520-0442(2001)014<4512:ISBTAA > 2.0.CO;2.

Hurrell, J. W., 1995: Decadal trends in the North-Atlantic Oscillation-regional temperatures and precipitation. Science, 269, 676-679, https://doi.org/10.1126/science.269.5224.676.

, Y. Kushnir, G. Ottersen, and M. Visbeck, Eds., 2003: An overview of the North Atlantic Oscillation. The North Atlantic Oscillation: Climatic Significance and Environmental Impact, Amer. Geophys. Union, 1-35, https://doi.org/10.1029/GM134.

Itoh, H., 2008: Reconsideration of the true versus apparent Arctic Oscillation. J. Climate, 21, 2047-2062, https://doi.org/10.1175/ 2007JCLI2167.1.

Johnson, N. C., and S. B. Feldstein, 2010: The continuum of North Pacific sea level pressure patterns: Intraseasonal, interannual, and interdecadal variability. J. Climate, 23, 851-867, https:// doi.org/10.1175/2009JCLI3099.1.

Jones, P., D. Lister, T. Osborn, C. Harpham, M. Salmon, and C. Morice, 2012: Hemispheric and large-scale land-surface air temperature variations: An extensive revision and updata to 2010. J. Geophys. Res., 117, D05127, https://doi.org/10.1029/ 2011JD017139. 
North, G. R., T. L. Bell, R. F. Cahalan, and F. J. Moeng, 1982: Sampling errors in the estimation of empirical orthogonal functions. Mon. Wea. Rev., 110, 699-706, https://doi.org/ 10.1175/1520-0493(1982)110<0699:SEITEO > 2.0.CO;2.

Poli, P., and Coauthors, 2016: ERA-20C: An atmospheric reanalysis of the twentieth century. J. Climate, 29, 4083-4097, https://doi.org/10.1175/JCLI-D-15-0556.1.

Quadrelli, R., and J. M. Wallace, 2004: A simplified linear framework for interpreting patterns of Northern Hemisphere wintertime climate variability. J. Climate, 17, 3728-3744, https:// doi.org/10.1175/1520-0442(2004)017<3728:ASLFFI >2.0.CO;2.

Rogers, J., and M. McHugh, 2002: On the separability of the North Atlantic Oscillation and Arctic Oscillation. Climate Dyn., 19, 599-608, https://doi.org/10.1007/s00382-002-0247-7.

Shi, N., and H. Nakamura, 2014: Multi-decadal modulations in the Aleutian-Icelandic low seesaw and the axial symmetry of the Arctic Oscillation signature, as revealed in the 20th century reanalysis. Tellus, 66A, 22660, https://doi.org/10.3402/ tellusa.v66.22660.

Smoliak, B. V., and J. M. Wallace, 2015: On the leading patterns of Northern Hemisphere sea level pressure variability. J. Atmos. Sci., 72, 3469-3486, https://doi.org/10.1175/ JAS-D-14-0371.1.

Sun, J., and B. Tan, 2013: Mechanism of the wintertime Aleutian low-Icelandic low seesaw. Geophys. Res. Lett., 40, 4103-4108, https://doi.org/10.1002/grl.50770.

Tan, B., L. Suo, and J. Huang, 2008: Variability of the coupling between surface air temperature and northern annular mode at various levels. Acta Meteor. Sin., 22, 277-283.

, J. Yuan, Y. Dai, S. B. Feldstein, and S. Lee, 2015: The linkage between the eastern Pacific teleconnection pattern and convective heating over the tropical western Pacific. J. Climate, 28, 5783-5794, https://doi.org/10.1175/JCLI-D-14-00568.1.

Thompson, D. W. J., and J. M. Wallace, 1998: The Arctic Oscillation signature in the wintertime geopotential height and temperature fields. Geophys. Res. Lett., 25, 1297-1300, https:// doi.org/10.1029/98GL00950.

, and - 2000: Annular modes in the extratropical circulation. Part I: Month-to-month variability. J. Climate, 13, 1000-1016, https://doi.org/10.1175/1520-0442(2000)013<1000: AMITEC $>2.0 . \mathrm{CO} ; 2$.
, and - 2001: Regional climate impacts of the Northern Hemisphere annular mode. Science, 293, 85-89, https://doi.org/ 10.1126/science. 1058958 .

Trenberth, K. E., and J. W. Hurrell, 1994: Decadal atmosphereocean variations in the Pacific. Climate Dyn., 9, 303-319, https://doi.org/10.1007/BF00204745.

Wallace, J. M., 2000: North Atlantic Oscillation/Annular Mode: Two paradigms-one phenomenon. Quart. J. Roy. Meteor. Soc., 126, 791-805, https://doi.org/10.1256/smsqj.56401.

- , and D. S. Gutzler, 1981: Teleconnections in the geopotential height field during the Northern Hemisphere winter. Mon Wea. Rev., 109, 784-812, https://doi.org/10.1175/1520-0493 (1981) $109<0784$ :TITGHF $>2.0$.CO;2.

— and D. W. J. Thompson, 2002: The Pacific center of action of the Northern Hemisphere annular mode: Real or artifact? J. Climate, 15, 1987-1991, https://doi.org/10.1175/1520-0442 (2002)015<1987:TPCOAO > 2.0.CO;2.

Wang, L., and W. Chen, 2010: Downward Arctic Oscillation signal associated with moderate weak stratospheric polar vortex and the cold December 2009. Geophys. Res. Lett., 37, L09707, https://doi.org/10.1029/2010GL042659.

,-- , W. Zhou, J. Chan, D. Barriopedro, and R. Huang, 2010: Effect of the climate shift around mid 1970s on the relationship between wintertime Ural blocking circulation and East Asian climate. Int. J. Climatol., 30, 153-158, https://doi.org/ 10.1002/joc. 1876

- P. Xu, W. Chen, and Y. Liu, 2017: Interdecadal variations of the Silk Road pattern. J. Climate, 30, 9915-9932, https://doi.org/ 10.1175/JCLI-D-17-0340.1.

Wegmann, M., Y. Orsolini, E. Dutra, O. Bulygina, A. Sterin, and S. Brönnimann, 2017: Eurasian snow depth in long-term climate reanalyses. Cryosphere, 11, 923-935, https://doi.org/ 10.5194/tc-11-923-2017.

Yuan, J., B. Tan, S. B. Feldstein, and S. Lee, 2015: Wintertime North Pacific teleconnection patterns: Seasonal and interannual variability. J. Climate, 28, 8247-8263, https://doi.org/ 10.1175/JCLI-D-14-00749.1.

Zhao, H., and G. W. K. Moore, 2009: Temporal variability in the expression of the Arctic Oscillation in the North Pacific. J. Climate, 22, 3110-3126, https://doi.org/10.1175/ 2008JCLI2611.1. 